\title{
Intuitive Interface for the Exploration of Volumetric Datasets
}

\author{
Rahul Sarkar ${ }^{1}$, Chrishnika de Almeida ${ }^{1}$, Noureen Syed ${ }^{1}$, Sheliza Jamal ${ }^{1}$, Jeff Orchard ${ }^{2}$ \\ ${ }^{I}$ Department of Electrical and Computer Engineering, University of Waterloo \\ ${ }^{2}$ David R. Cheriton School of Computer Science, University of Waterloo
}

\begin{abstract}
Conventional human-computer interfaces for the exploration of volume datasets employ the mouse as an input device. Specifying an oblique orientation for a crosssectional plane through the dataset using such interfaces requires an indirect approach involving a combination of actions that must be learned by the user. In this paper we propose a new interface model that aims to provide an intuitive means of orienting and translating a crosssectional plane through a volume dataset. Our model uses a hand-held rectangular panel that is manipulated by the user in free space, resulting in corresponding manipulations of the cross-sectional plane through the dataset. A basic implementation of the proposed model was evaluated relative to a conventional mouse-based interface in a controlled experiment in which users were asked to find a series of targets within a specially designed volume dataset. The results of the experiment indicate that users experienced significantly less workload and better overall performance using our system.
\end{abstract}

\section{INTRODUCTION AND RELATED WORK}

Many applications that make use of 3D visualization involve the formation of a volume dataset through interpolation within sets of 2D images. Notable examples include clinical imaging modalities such as magnetic resonance imaging (MRI) and computed tomography (CT), which rely on software construction of a volume from 2D slice acquisitions. The analysis of constructed volumes for diagnostic and therapeutic purposes generally involves a search for features of interest, and is usually conducted by viewing and manipulating one or more cross-sectional planes through the volume. Computing systems used for these applications feature software interfaces which provide orthogonal cross-sectional planes in the standard axial, sagittal, and coronal views through the imaged volume. The translation of these planes along the standard axes is an action well suited to mouse scrolling, where an upwards or downwards scroll respectively correspond to movement along a given axis in one direction or another. However, clinically relevant features often exist at orientations that are oblique with respect to the standard views. Support for interaction with oblique cross-sectional planes is often provided through the mouse by mapping combinations of button clicks and mouse motions to translation and rotation actions, requiring cognitive effort on the part of the user to learn and apply these combinations. Most commercial interfaces for dealing with volumetric data almost universally use the mouse as an input device, providing software tools for 3D manipulation using the mouse. An intuitive physical interface capable of providing 3D input could have significant utility towards improving user experience and reducing the workload associated with exploratory activities when compared with this mouse based interface.

There has been a great deal of research in 3D user interfaces to date, resulting in the development of a wide variety of systems in research settings. Most of the recent advances in 3D interface design for exploring volumetric data have been inspired by surgical planning applications, resulting in the creation of several application-specific interfaces that each correspond to a particular anatomical or surgical domain $[1,3,4,5,6]$. All of these studies made use of 3D user interfaces that were rated as being useful by the test users, usually surgeons. Yet despite the demonstrated effectiveness of interfaces such as these, 3D interfaces are still rare in both clinical settings and general computing systems. Balakrishnan [2] notes that most of us continue to communicate with our computing technology via primarily $2 \mathrm{D}$ interfaces, even when dealing with $3 \mathrm{D}$ data. There are likely several reasons behind this, including the high cost associated with many of these systems. Reitinger et al. [7] describe the main limitation of their system being used in clinical routine as hardware cost, since a virtual reality (VR) setup must be installed. Most of the above mentioned systems also make use of VR and are hence subject to similar costs due to the need for components such as rendering workstations. Due to such practical considerations, an interface that can be used with a standard workstation is may be more widely useful. Furthermore, all of the mentioned systems were developed for specific application domains, with tools and props customized to these applications. The abstraction of the 
problem domain to the exploration of any volumetric dataset presents the opportunity to create a general interface device that could have widespread applicability. While lacking some of the finer control points of the applicationspecific interfaces, such an interface could offer a similar character of generality that makes the mouse such a widely used two dimensional input device.

We present an interface model intended to be intuitive and versatile. Our model uses a hand-held rectangular panel that is manipulated by the user in free space, resulting in corresponding manipulations of the cross-sectional plane through the dataset. In the next section, we will describe a basic implementation of this model that using an electromagnetic tracking system, a minimal number of sensors coils, and a standard workstation, allowing us to evaluate the model with respect to a mouse-based interface. We describe a user study in which participants were asked to find a series of targets by manipulating a cross-sectional plane in a 3D image using both a mouse-based interface and the hand-held device interface. We then conclude with a description of the results and plans for future work.

\section{IMPLEMENTATION}

The components of the interface system that we designed include the hand-held panel, a magnetic motion tracking system, a PC and a standard display. The motion of the panel is tracked within the magnetic field generated by the motion tracking system. Two sensor coils are attached to diagonally opposite corners of the panel, and the motion tracking system measures five degree-of-freedom (5 DOF) position and orientation information for each coil. The PC polls the motion tracking system and retrieves positional data using application-programmer interface (API) calls. Visualization software converts the data for each sensor coil from the coordinate system of the motion tracking system to a standard graphical coordinate system and calculates the position of a third corner point based on the position and orientation of the two sensor coils and the known dimensions of the panel. The three points are then used to prescribe a cross-section through a previously loaded volume in the visualization software.

Figure 1 shows a high level overview of the system in use. The user moves the panel in the free space bounded by the motion tracking volume produced by a field generator (not shown). The motion data from the attached sensor coils are transmitted via sensor interface units to the control unit of the motion tracking system. The control unit relays the data to the $\mathrm{PC}$ through an RS-232 interface. Once the system software translates this data into a visualization plane in the volume dataset, the plane appears on the display, and the user continues to adjust the motion of the device based upon the observed plane.

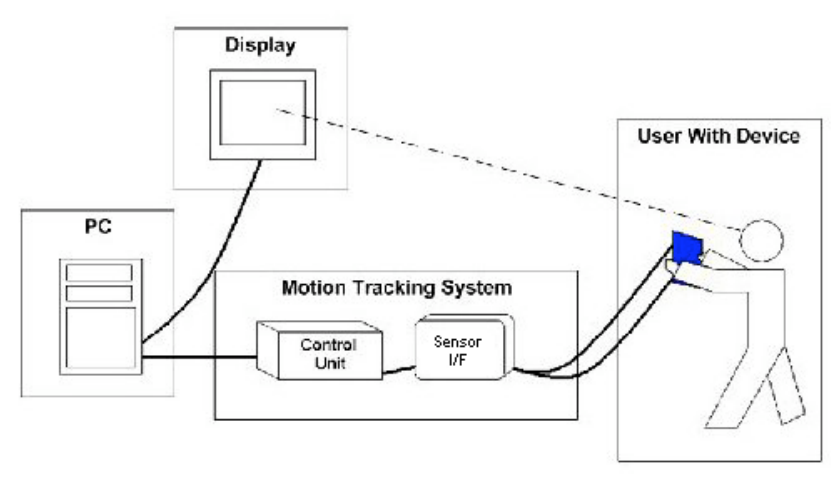

Figure 1. High-level overview of the system

Our implementation makes use of the Aurora tracking system from NDI (Northern Digital Inc., Waterloo, ON, Canada) for tracking the motion of hand-held panel. This tracking system uses electromagnetic measurement to track sensor coils within the scope of a defined measurement volume. The field generator produces an electromagnetic field with a characterized measurement volume of $50 \mathrm{~cm} \mathrm{x}$ $50 \mathrm{~cm} \times 50 \mathrm{~cm}$, projecting outward from one side of the generator. When the field generator is placed on a table across from roughly across from the upper torso of a seated user, the bounds of the measurement volume are comfortably within the reach of most users. System control and measurement data are accessible through a NDI Combined Polaris and Aurora Application Programmers' Interface, and communication between Aurora and the PC is done via a standard RS-232 port.

The hand-held panel is composed of a firm cardboard material and measures $25 \mathrm{~cm} \times 21 \mathrm{~cm} \times 0.5 \mathrm{~cm}$. The panel is comfortably gripped using either one or two hands, and is easily moved to arbitrary positions and orientations within the measurement volume. It can also be extended to the outer boundaries of the measurement volume with relative ease. Figure 2 shows the handheld panel with tracking tools attached. The panel uses two sensor tools attached to diagonally opposite corners. Each tool has a single 5 DOF sensor embedded within it, and is able to report 3D position coordinates with respect to the tracking volume, as well as orientations along any axis other than rotation about its longitudinal axis. The decision to use two sensors to measure corner points of the panel was motivated by the need to provide three points (or two lines) to define the plane of our desired cross-section, while trying to minimize the number of sensor tools needed. With two points directly measured in diagonally opposite corners, a third corner point along the longitudinal axis of either measured point can be calculated using the position information of that point, along with the combination of 5 DOF orientation information and the known distance between the measured point and the calculated point. 


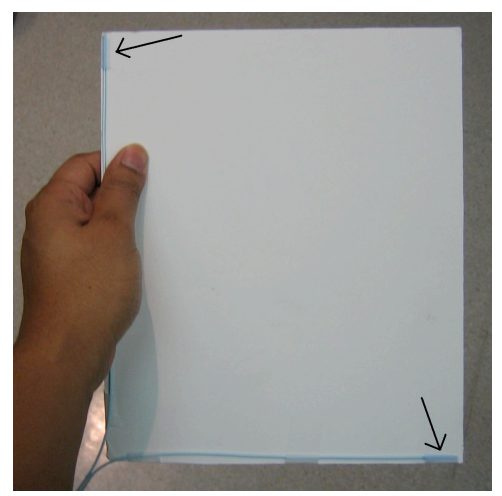

Figure 2. The Hand-held Panel (sensor coil positions indicated)

Positional coordinates reported by the tracking system are with respect to the measurement volume coordinate system, which differs from that of traditional graphical coordinate systems in visualization applications. The interface uses a software mapping to convert between coordinate systems, and the bounds of each axis are also linearly scaled to the dimensions of the 3D visualization. Orientation information from each sensor coil is retrieved from the tracking system in normalized quaternion form, which is then converted to rotation matrices using governing equations as described in [6]. The orientation information is with respect to the coordinate system of each sensor coil, which defines its longitudinal axis to be the zaxis.

The coordinates of the third corner point are calculated in order to enable the definition of the cross-sectional plane using lines that extend from the third point to each of the two measured points. The distance between one sensor coil and the corresponding corner point along its longitudinal axis was previously measured and is denoted L. In performing the calculation, the position of one of the sensor coils is assumed to be the origin, and the position of the missing point is assumed to be offset from the origin by a distance of $\mathrm{L}$ along the negative $\mathrm{z}$-axis with respect to the coordinate system of the coil. The rotation matrix $\mathrm{R}$ describing the orientation of the sensor coil is multiplied with the assumed position of the missing point, placing it at the correct point in space relative to the assumed sensor coil position at the origin. The translated position coordinates for the sensor coil are then added to the rotated position of the missing point, shifting the point to the correct absolute location within the measurement volume. This process is repeated to obtain a second position calculation using the data from the second sensor coil, and the final position values in each axis are averaged between the two sets, providing some error correction in the case of minor shifts in either coil. Figure 3 depicts this process using the second sensor coil position (x2, y2, z2) to calculate the missing third point $(\mathrm{x} 3, \mathrm{y} 3, \mathrm{z} 3)$.
1)

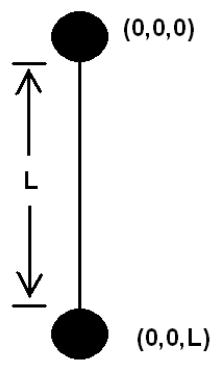

2)

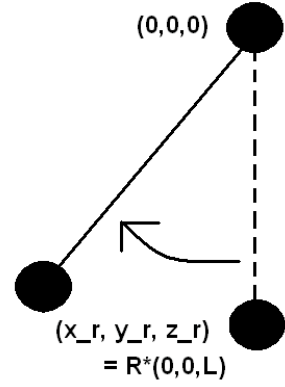

3)

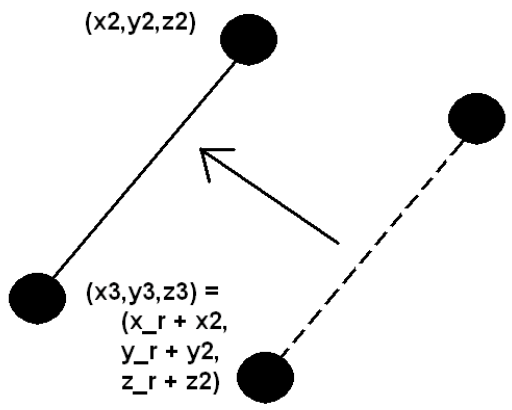

Figure 3. Calculation of the third point

All serial communication between the PC and Aurora was implemented in $\mathrm{C}++$ using the NDI Combined API for communication. Methods were written to initialize the system and ports, and poll the system for position and orientation information for each sensor coil. Coordinate system transformations, as well as conversion between quaternion and rotation matrix formats, were also implemented in C++. In order to support error handling in situations where either sensor coil is moved outside of the volume or the system undergoes temporary electromagnetic interference, additional pre-processing of the data was implemented to reject any samples that did not include valid coordinates for every axis and sensible orientation information. The methods were compiled under Windows $\mathrm{XP}$ and packaged as a dynamic-link library (DLL).

Visualization software was written in Python 2.3 using VTK 4.1 (www.vtk.org) using Atamai 1.0 (www.atamai.com) classes. The Atamai classes include support for the rendering of cross-sectional planes in the standard axial, sagittal, and coronal views as well as oblique planes. These classes all support mouse interaction through click and drag translation, and the oblique plane class further supports full plane rotation through additional click and drag methods. In order to add support for our hand-held panel, we used the SWIG interface compiler (www.swig.org) to generate Python wrappers for the C++ methods that communicate with Aurora and perform data pre-processing. The conversion between coordinate systems and calculations for specifying the plane were also implemented in Python. 


\section{USER STUDY}

We conducted a study to compare the general usability of our hand-held panel interface to that of a standard mousebased interface. Since the intended applications of our interface are to allow for effective and intuitive exploration of volume datasets, we were also interested the performance of users when finding regions of interest within a volume using each interface. To this end, we designed an experiment in which each participant was asked to find a series of clear targets within a 3D image of a specially designed volume. The completion times for each target acquisition were measured, and participants completed surveys regarding the workload in using each interface as well as their overall experience.

We hypothesized that completion times for the tasks assigned would be reduced when using the panel interface since the cross-sectional position and orientation is updated with the continuous motion of the panel, whereas the mouse based interface often requires composite actions to achieve the same cross-sectional prescriptions. We expected this difference to be more pronounced in the case of oblique targets when compared with axial targets, since oblique prescriptions generally require combinations of rotations in addition to basic translations. Workload factors of interest for the tasks used in the experiment were mental demand, performance, physical demand, frustration and effort. For assessing participant workload in completing the tasks, a software implementation of the NASA Task Load Index (NASA-TLX) workload rating was used [7]. At the conclusion of the experiment, participants completed a survey indicating which interface they preferred in completing the tasks, and which they found more intuitive for cross-section control.

The independent variables were the interface used and type of target. We chose two target types: axial targets, and oblique targets. For axial targets, the desired crosssectional target plane was situated at a particular depth along the axis normal to the axial view in the volume image. These target planes could be acquired without any need to rotate the cutting plane. For oblique targets, the plane was situated at some angle that was oblique to the standard views, requiring both rotation and translation operations from the default axial orientation. Our trials involved two different axial targets, and two different oblique targets. Each participant was asked to find one axial target and one oblique target using each interface, for a total of four targets. The participants were randomly assigned to one of two groups, and each of the groups was assigned different axial and oblique targets following an independent-measures design in order to eliminate the effects of target location learning by the participants. The order in which the targets were presented was based on a 4x4 Latin Square design for each group, where one dimension represented the target and the other dimension represented the four participants in the group, for a total of eight participants. Participants were undergraduate and graduate students from a variety of disciplines. The vast majority of participants reported their degree of computer usage as moderate to high, and their degree of experience with graphics/visualization software as minimal. For the test volume used in our experiments, we used a DICOM dataset of a specially constructed target set acquired using MRI. The target set included certain structural shapes and engravings intended to serve as clear target markers in the volume image acquired using MRI. Target markers were strategically placed at a variety of orientations. Figure 4 shows the targets that participants were asked to find during the experiment. Two of the four targets ( $a$ and $b$ ) were along planes that were roughly parallel to one of the axial views, and the other two targets (c and d) were along planes with orientations that were oblique with respect to the standard views.

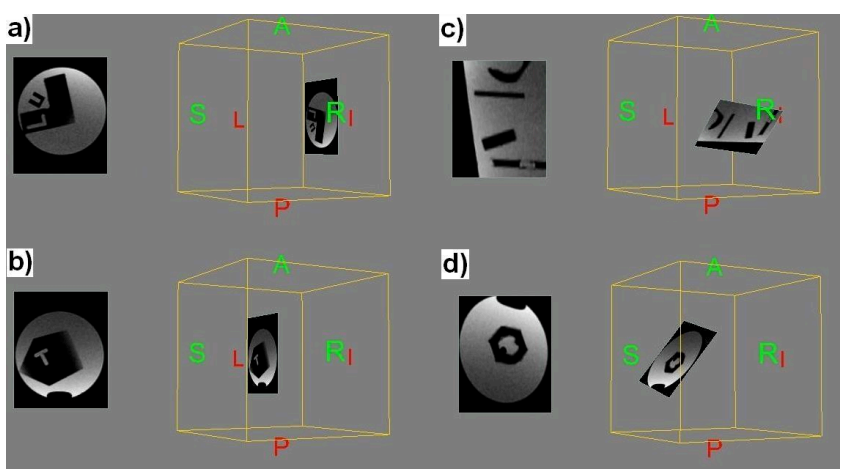

Figure 4. Axial ( $a$ and $b$ ) and oblique ( $c$ and $d$ ) targets acquired by participants.

The experiment was conducted on one participant at a time. Before beginning the experiment, each participant underwent a brief orientation session during which the general nature of the study and the experimental tasks were explained. They were then instructed on the use of the visualization software using both the mouse-based interface and the panel interface. These instructions were also presented in written form, and the participant was given the opportunity to ask for clarification. Once the experiment began, a target was presented on screen and the participant used the assigned interface to acquire the target within the volume image. This was done under the supervision of a coordinator who was present to enforce consistency in environmental factors between trials. Each participant was timed in their completion of each target task, and there was a maximum timeout of five minutes for the acquisition of each target. After completing the second task for each assigned interface, the participant was asked to complete the NASA-TLX workload rating. Once all tasks were completed, the trial concluded by having the participants 
complete a questionnaire regarding their overall preferences between the interfaces.

\section{Results}

Completion times for all tasks acquisitions using each interface were measured. Figure 5 shows the average completion times for axial targets, with error bars indicating one standard deviation. Average completion times for axial target acquisition was comparable between the two interfaces, with no statistically significant difference observed ( $\mathrm{p}=0.195)$. Average completion times were higher using the mouse interface for three of the four targets, with the exception being the first axial target (target b in Figure 4). This observation can likely be explained in part by the close proximity of the first axial target to the default starting position of the cross-sectional plane.

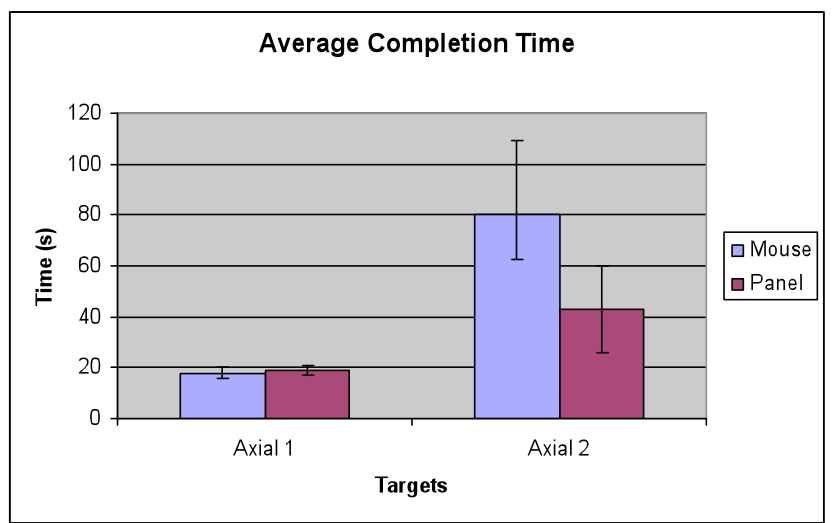

Figure 5. Average completion times for axial targets using each interface

The average completion times for the oblique targets are summarized in Figure 6. The average completion times for each target acquisition were markedly lower using the panel interface. We were able to show a statistically significant difference in completion times for the oblique targets $(p=0.0047)$ based on an alpha-level of $p=0.05$

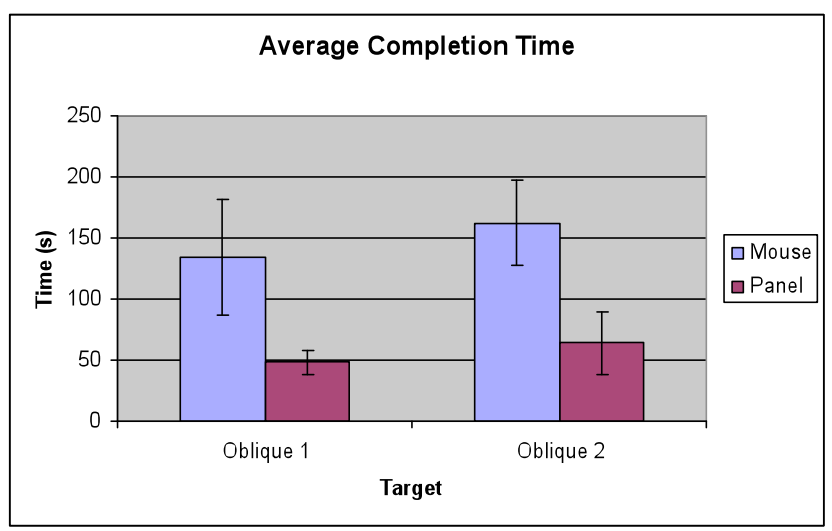

Figure 6. Average completion times for oblique targets using each interface

Participant responses to the NASA-TLX rating for each interface indicated lower average values for each workload factor besides physical demand when rating the panel interface. We were able to identify statistically significant differences for mental demand $(\mathrm{p}=0.041)$, effort $(\mathrm{p}=0.018)$ and frustration $(\mathrm{p}=0.010)$, with lower average workload values for the panel interface, as well as a statistically significant difference for physical demand $(p=0.003)$ with a lower average value for the mouse interface. Figure 7 shows the average workload values for each of these factors with error bars indicating one standard deviation.

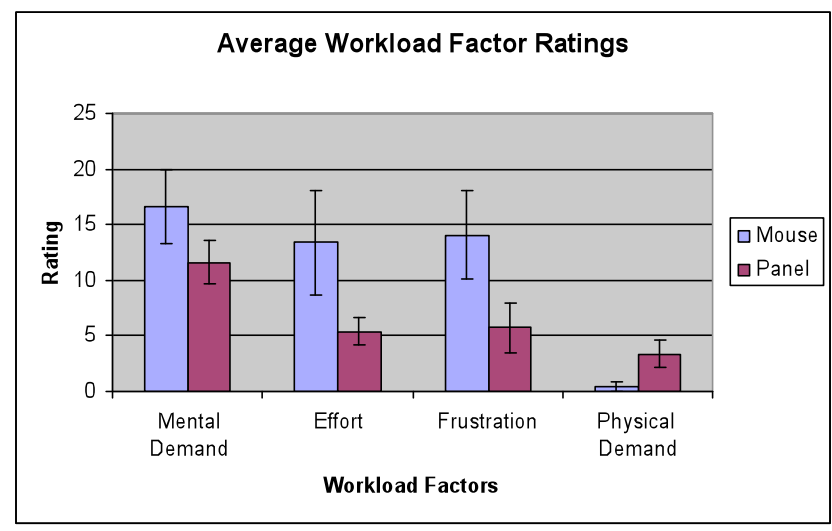

Figure 7. Average workload factor ratings for target acquisition using each interface

The higher average physical demand rating for the panel interface is statistically significant, but is still low in absolute terms when compared to ratings for other factors. The lack of significant difference in workload due to performance $(p=0.439)$ may be the result of statistically similar completion times for the acquisition of three of the four targets. The results for performance ratings may have also been affected by the phrasing of related survey questions. Several participants expressed confusion regarding questions relating to performance and temporal demand, and asked for clarification while completing the survey.

Figure 8 shows the average overall workload rating for each interface. We observed statistically-significant lower average overall workload ratings for the panel interface $(p=0.021)$. 


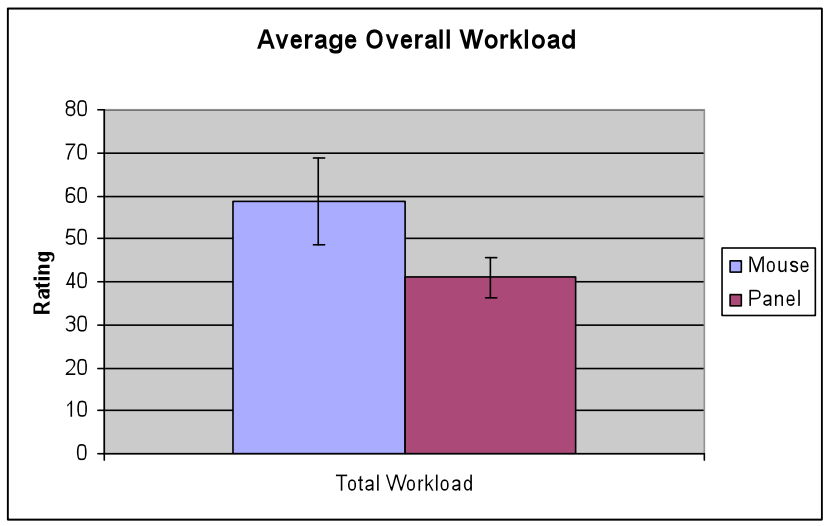

Figure 8. Average overall workload ratings for target acquisition using each interface

Responses to the overall experience questionnaire indicated that six of the eight participants preferred the use of the panel interface to the mouse for completion of the tasks, and all participants found the panel interface more intuitive for the control of the cross-sectional plane.

\section{Conclusions}

In this paper, we proposed the use of a new 3D interface model for the exploration of volumetric datasets. With the aim of designing an interface that takes advantage of proprioceptive abilities while remaining generically applicable, the model makes use of a hand-held panel that models the cross-sectional plane of interest, as opposed to props that model application-specific features. We described a basic implementation of this model, along with a user study that evaluated its usefulness against that of a mouse-based interface.

From the results of the study, the performance using each interface is comparable for acquiring targets in a standard axial view, but can be significantly improved for acquiring oblique targets by using the panel interface. In practice, features of interest can exist at arbitrary locations and orientations within the volume during exploratory activities, emphasizing the importance of the improved oblique exploration offered by the panel interface.

Based upon lower NASA-TLX overall workload ratings for the panel interface, as well as lower ratings across several factors including mental demand, effort, and frustration, we conclude that the use of panel interface for completing the task involves significantly less workload than the mouse-based interface. While the physical demand of using the panel interface was found to be significantly higher than that of using the mouse-based interface, the physical demand was found to be a very low contributor to the overall workload experienced for both interfaces. From the results of the overall experience questionnaire, we conclude that panel interface is generally preferred to the mouse-based interface for feature localization tasks such as the target acquisitions in the user study, and provides more intuitive control of the crosssectional plane.

Finally, given that the panel interface model can be applied to any type of volumetric data exploration, while involving implementation costs significantly less than comparable interfaces that make use of VR immersive environments, we believe that this model has the potential for widespread use in volumetric data exploration.

\section{FUTURE WORK}

We are currently examining alternative implementations for the interface model, including the use of optical tracking using infrared sensors and triangulation, in order to further reduce the associated costs. In addition, we are exploring the possible application of this interface model towards real-time scan plane control in MRI, where the absolute position of the panel with respect to the reference could be linked to the position of the imaging plane in the target.

\section{ACKNOWLEDGMENT}

We would like to thank Ali Abharisa and Dingrong Yi from Sunnybrook Research Institute for providing the phantom volume image used for target acquisition in the user study.

\section{REFERENCES}

[1] A.F. Ayoub, D. Wray, K.F. Moos, P. Siebert, J. Jin, T.B. Niblett, C. Urquhart, and P. Mowforth. Three-dimensional modeling for modern diagnosis and planning in maxillofacial surgery. Int. J. Adult Orthod.Orthognath. Surg, 11:225.233, 1996.

[2] R. Balakrishnan. Why Aren't We Using 3D User Interfaces, and Will We Ever? IEEE 3DUI 2006, viii-viii, 2006.

[3] H.S. Byrd and P.C. Hobar. Rhinoplasty: a practical guide for surgical planning. Plast. Reconstr. Surg., 91:642.654, 1993.

[4] E.Y.S Chao and F.H. Sim. Computer-aided preoperative planning in knee osteotomy. Iowa Orthop. J., 15:4.18, 1995.

[5] S. Haasfeld, J. Zoller, F.K. Albert, C.R. Wirtz, M. Knauth, and J. Muhling. Preoperative planning and intraoperative navigation in skull base surgery. J. Cranio-Maxillofac. Surg., 26:220.225, 1998.

[6] K. Hinckley, R. Pausch, J.H. Downs, D. Proffitt, and N.F. Kassell. The Props-Based Interface for Neurosurgical Visualization. Stud. Health Technol Inform, 39:552-562, 1997.

[7] B. Reitinger, D. Schmalstieg, A. Bornik, and R. Beichel. Spatial Analysis Tools for Virtual Reality-based Surgical Planning. IEEE 3DUI 2006, 37-44, 2006. 